\title{
La distribución social del ingreso. El caso de Uruguay, 2008-2014*
}

\author{
Social income distribution. The Uruguayan case, 2008-2014
}

\section{Jorge Notaro**}

\footnotetext{
* Economista. Integrante del Sistema Nacional de Investigadores de la ANII. Investigador del Núcleo Interdisciplinario «Pensamiento Crítico en América Latina y Sujetos Colectivos» de la UDELAR. $\bowtie$ jnotaro@netgate.com.uy
}

RECIBIDO: [13.3.2018]

ACEPTADO: [4.6.2018]

\begin{abstract}
Resumen
El objetivo principal del artículo es diseñar una metodología de análisis de la distribución del ingreso que se define como social y utilizarla para responder a la pregunta ¿Cómo se distribuyen los ingresos entre grupos sociales en el Uruguay a principios del siglo XXI? El principal resultado es la estabilidad con ligeras fluctuaciones. Durante los últimos tres años del período, los ingresos del capital se ubicaron en 16.100 millones de dólares anuales, los de obreros y empleados en 9.759,2 y los de pasivos en 4.740,6. Estos resultados son originales, así como la identificación de los ingresos del capital con información tributaria que permitió superar la insuficiente captación de la Encuesta Continua de Hogares (ECH) del Instituto Nacional de Estadística (INE), la inclusión de los ingresos del capital extranjero y la desagregación de la masa salarial identificando los ingresos de obreros y empleados.
\end{abstract}

Palabras clave: económicas, distribución del ingreso, metodología, Uruguay.

JEL: B 41, B 50, E 01, E 25, R 20 


\begin{abstract}
How is income distributed among social groups in Uruguay at the beginning of the 21st century? To answer this question, the article describes a methodology designed to analyze the so-called "social income". The main outcome is stability, with slight fluctuations. During the last three years of the period considered, capital inflows stood at 16,100 million dollars per year, 9,759.2 of which accounted for workers and employees and 4,740.6 for retirees. These findings are novel, as well as the procedure used to identify capital inflows through tax information, (which allowed to overcome the insufficient data gathering of the National Statistics Institute's Continuous Household Survey), the inclusion of foreign capital inflows and the disaggregation of the wage bill to identify the incomes of workers and employees.
\end{abstract}

Key words: economics, income distribution, methodology, Uruguay.

JEL: B 41, B 50, E 01, E 25, R 20.

\title{
Introducción
}

El objetivo principal del artículo es diseñar una metodología de análisis de la distribución del ingreso que se define como social y utilizarla en el caso uruguayo para responder a la pregunta ¿Cómo se distribuyen los ingresos entre grupos sociales en el Uruguay a principios del siglo XXI? La distribución social es la estimación de los ingresos de grupos sociales que están constituidos por personas insertas de la misma forma en la actividad económica, que no se limita a tres clases sociales y tres formas de ingresos, sino que recupera la correspondencia entre las categorías de ingresos y la estructura social de la economía política.

Se revisaron los principales aportes teóricos como soportes del marco conceptual, y como componentes de este se definieron categorías, se evaluaron fuentes de información y se diseñaron procedimientos para construir indicadores (segunda sección).

Se analizaron exhaustivamente las investigaciones sobre Uruguay, poniendo el foco en la metodología y las fuentes utilizadas. Se diferenciaron los trabajos iniciales del período 1965 a 1984, el cambio de enfoque posterior, el reconocimiento de las limitaciones de las encuestas de hogares (EH), los intentos de superación con información tributaria y los que se apartan de la orientación predominante. Por último se estableció un balance que justifica el posible aporte del presente artículo (tercera sección). Se estimaron resultados para Uruguay que permiten concluir que en el período analizado predominó la estabilidad con ligeras fluctuaciones en la participación de los ingresos de los grupos en el producto interno bruto a precios de mercado PIB pm (cuarta sección). 
La originalidad del presente artículo se manifiesta en la estimación de la distribución del total de ingresos generados entre grupos sociales, la identificación de los ingresos del capital con información tributaria que permitió superar la insuficiente captación de la Encuesta Continua de Hogares (ECH) del Instituto Nacional de Estadística (INE), la inclusión de los ingresos del capital extranjero y la desagregación de la masa salarial para identificar los ingresos de obreros y empleados.

\section{Principales antecedentes teórico-metodológicos y marco conceptual}

Se revisaron los principales aportes teóricos desde los orígenes de la preocupación por el tema hasta los estudios recientes abarcadores de América Latina, como soportes del marco conceptual que se presenta al final de este punto.

Para Quesnay, los ingresos se correspondían con la estructura social: para los agricultores, en forma de excedente, y para los propietarios, en forma de rentas, impuestos y diezmo; para los trabajadores sin propiedad, en forma de salarios; para los comerciantes, en forma de ganancias, y para los «financieros», en forma de intereses de créditos o de deuda pública (1758/1974, p. 73).

Los fundadores de la economía política, Adam Smith, David Ricardo y Karl Marx, analizaron la distribución del ingreso entre tres clases sociales principales, pero no únicas: los terratenientes, los capitalistas y los trabajadores. Cada una percibía un ingreso por su forma de participar en el proceso económico: la renta, las ganancias y los salarios. Smith (1789/1904), en el «Book I of the Causes of Improvement», ${ }^{1}$ consideró la distribución entre «different Ranks of the People»; la renta de la tierra resultaba de la propiedad privada de esta, y señaló situaciones en las que dos o tres formas de ingresos podían ser percibidas por una misma persona. Ricardo, en el prólogo a la primera edición de Principles of Political Economy and Taxation, destacó que el producto «is divided among three classes of the community; namely, the proprietor of the land, the owner of the stock or capital necessary for its cultivation, and the labourers by whose industry it is cultivated» (1821/2001, p. 5). Marx señaló: «The owners merely of labour-power, owners of capital, and land-owners, whose respective sources of income are wages, profit and ground-rent, in other words, wage-labourers, capitalists and land-owners, constitute then three big classes of modern society based upon the capitalist mode of production» (1894).

1 Las citas corresponden a la edición de Cannan de 1904 (Methuen \& Co.) de la quinta edición de An Inquiry into the Nature and Causes of the Wealth of Nations. En ella las referencias a números de página fueron sustituidas por parágrafos numerados. 
Marshall distinguió el análisis de los ingresos «desde el punto de vista de los negocios privados» y desde «el punto de vista social» (1957, p. 63). Señaló que «el problema de la distribución del ingreso es mucho más difícil de lo que pensaron los primeros economistas» (1957, p. 420) y agregó como condicionantes la relación entre la oferta y la demanda de los agentes de producción (1957, p.429). Conservó algunas categorías de la economía clásica y cuestionó otras, por lo que se lo puede considerar el comienzo de la transición.

Jevons inició el camino hacia un nuevo enfoque: «El énfasis se colocó en los consumidores, sus cálculos de placer y dolor, por lo que la distribución se determina en el mercado y los factores de producción perciben ingresos según su productividad marginal» (Dobb, 1988, p. 194). Dobb criticó el enfoque que «al reducir la distribución a la formación de precios de los servicios o factores productivos dio como resultado la exclusión de las circunstancias sociales de los oferentes (o de los grupos sociales vinculados a la oferta) de estos "servicios" hasta el punto de perder de vista la existencia misma de estos individuos».

Un nuevo cambio de énfasis se produjo en los años treinta; «la materia de los estudios sobre el ingreso nacional, tanto en su aspecto cuantitativo como conceptual, recibió gran impulso» (Roll 1967, p. 470). Kusnetz $(1933,1941)$ definió y estimó las categorías que integran el ingreso nacional, considerando los salarios, las utilidades, el ingreso de los empresarios y los intereses. El predominio de este enfoque generó la crítica de Stiglitz: «Although the recent literature has abounded with alternative theories of distribution of income among factors of production, there have been few attempts to develop a theory of distribution of wealth and income among individuals» $(1969$, p. 384).

Los principales aportes desde el fin del siglo XX se encuentran en los manuales sobre distribución del ingreso editados por Atkinson y Bourguignon $(2000,2015)$ y sobre desigualdad, enfoque predominante en los últimos años (Atkinson, 1989; Nolan, Salverda y Smeeding, 2011). Se centran en la distribución personal en países capitalistas desarrollados y hacen alguna referencia a países de menor desarrollo (Atkinson, 1989, p. 28-31).

El cambio de enfoque se fundamentó en las limitaciones señaladas a la economía clásica, como los cambios en las estructuras social y económica (Atkinson, 1989, p. 220221), la heterogeneidad de las clases capitalista y trabajadora (Atkinson, 1989, p.200), los salarios de los directivos y gerentes que deberían considerarse ingresos del capital (Glyn, 2009, p.112), la posibilidad de que una persona perciba ingresos de distinto origen, la dificultad de desagregar los ingresos de los trabajadores por cuenta propia entre capital y trabajo (Atkinson, 1989, p. 201; Glyn, 2009, p. 110), las utilidades no distribuidas separadas en el Sistema de Cuentas Nacionales (SCN) y el papel del Estado en la redistribución (distribución secundaria en el SCN). 
En los últimos años son muy pocas las investigaciones de la distribución del ingreso con un enfoque de economía política adaptado a los cambios en las estructuras social y económica. Wolff y Zacharias (2007) estimaron los ingresos del capital y de los diversos grupos de trabajadores en los Estados Unidos en 1989 y 2000; Wodtke (2014) estimó los ingresos del capital y diferenció los directivos y gerentes en los Estados Unidos entre 1983 y 2010, considerando otras condicionantes como el nivel educativo o el color de la piel; Mohun (2016) diferenció tres clases, según tuvieran ingresos del capital o del trabajo con o sin responsabilidades de dirección.

Algunos trabajos relacionan la distribución del ingreso con las clases sociales, pero definen estas en función de los niveles de ingresos y se apartan del enfoque de la economía política, como Avaar (2008); Muntaner, Lynch y Oates (1999); Chai y Kaus (2012); Blanden, Gregg y Macmillan (2013); Curtis y Andersen (2015). Barrett y Esteban (2005) caracterizaron los grupos sociales de acuerdo a criterios socioculturales como la religión y la etnia.

En América Latina (AL) las investigaciones sobre distribución del ingreso se orientaron casi exclusivamente a la distribución personal, utilizando información de las EH, y excepcionalmente señalaron limitaciones en el registro de algunos ingresos. Entre los que analizaron la evolución de la desigualdad de ingresos personales en AL con índices de Gini se destacan el De Ferranti et al. (2003), López-Calva y Lustig (2010), Lustig, Pessino y Scott (2013) y Gasparini, Cicowiez y Sosa Escuderol (2014). Entre los segundos, Székely y Hilgert (1999) observaron que las EH no logran registrar los ingresos de los sectores más ricos y como consecuencia se subestima la desigualdad que se puede medir; Morley señaló que las EH subestiman el ingreso por concepto de utilidades (2000, p. 74), Cornia (2014) consideró que las EH subestiman los altos ingresos, corrigió los datos con información tributaria y calculó índices de Gini corregidos.

Alvaredo y Gasparini investigaron la desigualdad y la pobreza en el mundo en desarrollo, evaluaron fuentes e indicadores, identificaron diferencias entre regiones, así como las tendencias desde la década de los ochenta hasta principios del siglo XXI. Señalaron las limitaciones de las encuestas de hogares para captar los ingresos más altos y utilizaron también registros tributarios, y concluyeron que los altos ingresos pertenecían esencialmente a rentistas y propietarios de capital. Destacaron: «Results, even if fragmentary, confirm that incomes reported to the tax authorities are considerably higher than those captured by the surveys at the top» (2013, p. 19).

Como punto de partida del marco conceptual se plantea la interrogante a la que se trata de dar respuesta: ¿Cómo se distribuyen los ingresos entre grupos sociales en el Uruguay a principios del siglo XXI?

La respuesta requiere superar las críticas realizadas a los fundadores de la economía política y sobre la incapacidad de las EH para registrar los ingresos del capital, a fin de recuperar la relación entre los ingresos y la estructura social. Con estos objetivos se 
definieron categorías, se evaluaron fuentes de información y se diseñaron procedimientos de medida.

Se define como distribución social la estimación de los ingresos de grupos sociales que se delimitan con diversos criterios y están constituidos por personas que se insertan de la misma forma en la actividad económica, sin afirmar que constituyen clases sociales. Estos grupos «existen de hecho y no son meramente clasificaciones construidas», como destacó Terra (1983, p. 137), y se diferencian de los grupos de familias o de personas que solo existen como ideas; también Portes y Hoffman (2003) y Féliz, Fernández y López (2010) investigaron la distribución del ingreso entre grupos sociales delimitados por la categoría y el tipo ocupacional. Este enfoque se diferencia de la distribución factorial, así como de las estimaciones del SCN, porque reconoce la heterogeneidad de los trabajadores asalariados y los desagrega para identificar un grupo social homogéneo de obreros y empleados.

Se considera que el total de ingresos generados es el PIB pm, definido como ingresos primarios en el enfoque del ingreso del SCN. Se toma la definición del manual de la OCDE, «GDP is also equal to total income» (Lequiller y Blades, 2006, p. 28), que comprende más ingresos que las $\mathrm{EH}$-que se limitan a los que perciben los hogares residentes- La delimitación de los grupos sociales se apoya en el enfoque de la economía política clásica, pero no se limita a tres grupos y tres formas de ingresos para superar las críticas. Se diferencian los siguientes grupos sociales y categorías de ingresos:

a. Obreros y empleados, un grupo homogéneo de trabajadores asalariados o en relación de dependencia. Reconociendo la heterogeneidad de los asalariados, para identificarlos se separan como grupos, así como sus ingresos, los directores y gerentes de empresas, los que ocupan cargos políticos, las fuerzas armadas y los profesionales universitarios. La posibilidad de tener otros ingresos como utilidades, intereses o alquileres es importante entre los directores y gerentes que tienen capacidad de ahorro, pero para los obreros y empleados la magnitud es irrelevante en el total de los ingresos (Instituto de Economía [IECON], 1971).

b. Los del capital, que resultan de la propiedad. Se diferencian los propietarios de capital radicados en el país y los radicados en el exterior.

c. Los trabajadores que aplican su capacidad de trabajar sobre medios de producción de poca magnitud de su propiedad, a veces con familiares y transitoriamente con trabajadores asalariados. Sus ingresos corresponden a una forma de inserción en la actividad económica y tienen una referencia en la estructura social. En la EH se definen con la categoría ocupacional trabajadores por cuenta propia, y en el SCN sus ingresos se registran como ingresos mixtos. 
d. Los pasivos, un grupo social numeroso y organizado, que perciben ingresos en forma de transferencia del Estado. Su número fluctúa en torno al $50 \%$ de las personas que trabajan, y un indicador de su poder es el éxito en reformar la Constitución en 1989 mediante un plebiscito que incorporó un párrafo por el cual los aumentos de las pasividades de cada año serían, como mínimo, iguales al aumento del índice medio de salarios del año anterior.

e. El Estado, que en el PIB pm capta ingresos en forma de impuestos indirectos menos subsidios. Se agregan los impuestos y contribuciones a la seguridad social cuando los ingresos de los grupos sociales se miden después del pago de estos.

\section{La literatura sobre el caso uruguayo}

Se revisaron exhaustivamente las investigaciones sobre Uruguay poniendo énfasis en la metodología y las fuentes utilizadas. Se diferencian: a) los trabajos iniciales del período 1965 a 1984, b) el cambio de enfoque posterior, c) el reconocimiento de las limitaciones de las eh, d) los intentos de superación con información tributaria y e) los que se apartan de la orientación predominante. Por último, se establece un balance que justifica el posible aporte del presente artículo (f).

a. En la construcción del SCN por el Departamento de Investigaciones Económicas del Banco de la República Oriental del Uruguay (BROU) se definió el ingreso nacional como «la suma de remuneraciones de los empleados y obreros y de las utilidades y otras retribuciones netas del capital y del empresario recibidas en el país. Excluye por lo tanto, a las remuneraciones transferidas al exterior e incluye las recibidas del exterior» (Banco República [BROU], 1965). Se señaló la dificultad para estimar los ingresos del capital, «por cuanto el impuesto a la renta es de reciente aplicación en el país y con un marco limitado de captación». En «las remuneraciones brutas del sector capital-empresario» se diferenciaron la depreciación de activo fijo; los alquileres urbanos de viviendas y arrendamientos rurales; utilidades, intereses, rentas y alquileres netos de empresas y gobierno (BROU, 1965, p. 64 - 66). Se aclaró que «los ingresos de los pequeños propietarios, trabajadores independientes, profesionales por cuenta propia y miembros de familia sin remuneración quedan afectados al sector capital-empresa» (BROU, 1965, p. A61).

En el Plan Nacional de Desarrollo Económico y Social 1965-1974 se realizaron tres aproximaciones a la distribución del ingreso: por factores, por estratos de ingresos familiares y según la categoría socioprofesional del jefe de hogar. Excluido el aporte personal a la seguridad social, «los sueldos y salarios netos han constituido un $37 \%$ y un $39 \%$ del ingreso nacional bruto, en ambos períodos» (Comisión de Inversiones y Desarrollo Económico [CIDE], 1966, p. 215). Se establecieron hipótesis de menor y de mayor 
desigualdad para corregir las omisiones y asignar los ingresos. En la primera, el $20 \%$ de las familias de menores ingresos captaba un 4,6\% del total y el $10 \%$ de mayores ingresos captaba un 33,9\%; en la segunda, los porcentajes fueron 3,0\% y 45,8\% respectivamente (p. 216). Para la tercera aproximación se diferenciaron 12 categorías, se estimó el porcentaje del ingreso de cada una por familia y se señaló que no fue posible diferenciar los ingresos correspondientes al trabajo y a la propiedad (p. 219).

El Instituto de Economía (IECON) de la Facultad de Ciencias Económicas y Administración (FCEA) de la Universidad de la República (UDELAR) investigó la distribución por categoría ocupacional y concluyó que «las desigualdades y diferencias en los niveles de ingreso se explican fundamentalmente a partir de la categoría socioeconómica del perceptor», mientras que «la incidencia de la educación, la edad y el sexo es subsidiaria» (IECON, 1971, p. 97). «El ingreso medio de cada asalariado se constituye en un 95\% por el proveniente de la actividad principal. Esto a su vez indica una reducida importancia del ingreso de actividad secundaria o resultante de transferencias de la propiedad o del sistema de seguridad social» (IECON, 1971, p. 41). Comparando los ingresos de asalariados y patrones con iguales niveles de educación y edad, así como del mismo sexo, demostró que «La distribución del ingreso personal está determinada principalmente por la situación de cada perceptor respecto de la propiedad de los medios de producción» (IECON, 1971, p. 45).

Harberger y Wisecarver (1978) estimaron las tasas de retorno al capital entre 1967 y 1971, así como la participación de los ingresos del capital en el ingreso nacional, que se ubicó en torno al 32\%; con la misma metodología se revisó la información utilizada y se continuaron las series hasta 1978 (Banco Central del Uruguay [BCU], 1980).

Melgar analizó la información disponible y las limitaciones que esta imponía a los procedimientos e indicadores a utilizar (1981a) y luego investigó la evolución de la distribución personal del ingreso desde la década de los años sesenta (1981b). Melgar y Villalobos (1986) estimaron la omisión en la EH en comparación con el SCN y consideraron probable que en los tramos altos fuera alta y baja en los tramos de menores ingresos.

Terra analizó una «distribución social» estimando el ingreso medio y algunos indicadores de condiciones de vida para 14 categorías socioprofesionales. Estimó la omisión en $25,9 \%$ del total de ingresos, mínima en salarios pero relevante en los ingresos del capital, la corrigió reconociendo que, aunque ello implicaba algún grado de arbitrariedad, pero «la falta de corrección induciría ciertamente a error» (1983, p. 23). Finalizó su investigación con una «descripción morfológica de las clases sociales a partir de las categorías socio-profesionales de los estratos de ingresos» (1983, p. 155) y destacó que «al hablar de clases sociales nos referimos a grupos muy amplios, pero a grupos reales, que presentan una trama de interacciones y contenidos culturales específicos. Estos 
grupos existen de hecho y no son meramente clasificaciones construidas» (1983, p. 137).

b. Con el retorno a la democracia, en 1985, se inició una etapa en la que predominaron las investigaciones sobre la distribución personal utilizando diversos indicadores. Algunos trabajos realizaron una aproximación a lo que definieron como distribución funcional.

La mayor parte de las investigaciones aceptaron la información de la EH sin relativizaciones. Como antecedente, Bensión y Caumont (1979) investigaron los cambios en la distribución del ingreso familiar y funcional entre 1970 y 1976 con énfasis en el papel de la política económica. La Comisión Económica para América Latina y el Caribe (CEPAL) analizó la distribución del ingreso urbano entre los hogares, entre los perceptores y entre 19 grupos socioocupacionales de Montevideo en el período 1984-1988. Entre las conclusiones destacó: «El factor categoría cobra importancia en el período en la explicación de la desigualdad, lo que brinda indicios sobre las redistribuciones que pudieron haberse realizado con el aumento de los ingresos por otros conceptos, aparte de los salariales» (1989, p. 5).

Bucheli y Rossi (1994) investigaron la distribución del ingreso en Montevideo en el período 1984-1992 y no encontraron diferencias sustanciales en los índices de desigualdad calculados en el período (Gini y Theil). Melgar (1995) calculó el índice de Gini con información de la EH ordenando los hogares según su ingreso total y según el ingreso per cápita, y concluyó que la concentración del ingreso se redujo entre 1989 y 1993. Bucheli y Furtado analizaron el papel de las diversas fuentes entre 1986 y 1997, la distribución del ingreso en el período 1986-1997 (2001) y para el período 1998-2002 (2004) utilizando índices de Kernel y de Atkinson. Gradín y Rossi (2001) investigaron el papel de las jubilaciones y la heterogeneidad de los salarios. Perazzo, Robino y Vigna (2002) analizaron los impactos del sistema tributario sobre la distribución del ingreso y citaron como antecedente un trabajo de Bisogno y Grau (1989). Corrazzo y Sander (2005) investigaron la relación entre distribución del ingreso y crecimiento en la segunda mitad del siglo XX, construyeron algunos indicadores para el período previo a disponer de los datos de la ECH, citaron como antecedentes a Bértola $(2001,2005)$ y Ardente, Rossi y Díaz (2003) y recuperaron el aporte de Martínez (1981) en la estimación del excedente agropecuario. Grau y Lagomarsino (2002) examinaron los impactos de la estructura tributaria sobre la distribución del ingreso de los hogares por fuente y por decil. Marroig y Oreiro (2008) utilizaron microsimulaciones para descomponer las determinantes de los cambios en la distribución del ingreso de los hogares en el período 1991-2005. En el Informe de Desarrollo Humano en el Uruguay de 2007 se estimó la participación del trabajo y el capital en el PIB asignando a uno y otro parte de los ingresos de patrones y trabajadores por cuenta propia (Programa de las Naciones Unidas para el 
Desarrollo [PNUD], 2008). Bucheli y González concluyeron que «al menos de la descripción de los últimos treinta años, no puede extraerse una relación estable entre crecimiento y desigualdad» (2010, p. 339), tras medir la segunda con los índices de Gini y de Theil. Muinelo y Roca (2017) investigaron el impacto del gasto público sobre la desigualdad de ingresos en el país utilizando el índice de Gini. El INE calcula el índice de Gini con el ingreso por persona sin valor locativo para el total del país y los resultados muestran una tendencia descendente desde 0,455 en 2006 hasta un mínimo de 0,381 en 2014, un leve aumento a 0,386 en 2015 y un descenso en los dos años siguientes hasta 0,380 en 2017 (Instituto Nacional de Estadística [INE], 2018, p. 44).

Amarante y Vigorito (2011), así como De Rosa (2017), fundamentaron el cambio que llevó a abandonar el enfoque de la economía política clásica y analizar la distribución personal medida por el índice de Gini con información de la ECH, por la heterogeneidad entre quienes reciben ingresos del capital y del trabajo. Retomaron los argumentos de Atkinson y Glyn, sin tener en cuenta aportes que adecuaron el enfoque a los cambios en la sociedad y en la economía.

c. La preocupación por la subestimación de los ingresos de los hogares en la EH llevó a Grosskoff (1991), así como a Fuentes y Mendive (1996), a calcularla. Vigorito (1999) investigó la distribución entre los hogares urbanos, incorporó índices para analizar la desigualdad entre grupos y dentro de ellos, y concluyó que la distribución del ingreso en el Uruguay entre 1986 y 1997 se mantuvo estable. Advirtió sobre las implicaciones de la limitación de la EH para captar «los ingresos de los patrones y los ingresos del capital, lo que constituye una severa limitación en lo que respecta a los resultados del presente estudio» (1999, p. 8). Amarante y Carella (2000) utilizaron los porcentajes de subestimación por fuente de los anteriores, corrigieron la información de las EH para tres años (1991, 1993 y 1995) y concluyeron que «El índice de Gini es mayor en todos los años, tanto en Montevideo como en el interior, si se toma como referencia el ingreso corregido» (2000, p. 31).

d. Luego de la difusión de los libros de Atkinson (2007) y de Piketty (2013), que señalaron que la información fiscal registra mejor los altos ingresos que las EH (2013, p. 40), se realizaron varios trabajos sobre países de AL con información fiscal. Jiménez propuso «avanzar hacia el mayor aprovechamiento de las bases tributarias para fines estadísticos, con miras a obtener un mapa más adecuado de la desigualdad en América Latina» (2015, p. 11). Amarante y Jiménez investigaron los casos de Argentina, Colombia y Uruguay, y afirmaron: «Quizás la más importante de estas limitaciones la constituyan los problemas para captar adecuadamente los ingresos de la parte alta de la distribución» (2015, p. 21). Según estimaron, «El índice de Gini corregido, con la incorporación de los datos del 1\% más rico a partir de datos tributarios, resulta considerablemente superior al que surge de las encuestas de hogares en los tres países de la región analizados» (2015, p. 23). Gómez Sabaíni y Rossignolo analizaron las limitaciones de 
esta metodología y concluyeron que «los estudios realizados para identificar y evaluar la incidencia de los sectores de muy altas rentas en la distribución del ingreso de los países latinoamericanos son muy recientes, acotados en su alcance y limitados en cuanto a los resultados obtenidos y a las conclusiones que permiten sacar» (2015, p. 87).

En Uruguay, cuatro trabajos recientes utilizaron la información de recaudación de la Dirección General Impositiva (DGI) para estimar los «altos ingresos» que no registra la ECH. Burdín, Esponda y Vigorito (2015) y Burdín, De Rosa y Vigorito (2015) analizaron la distribución personal del ingreso utilizando los microdatos de los registros tributarios del impuesto a la renta de las personas físicas (IRPF) y del impuesto de asistencia a la seguridad social (IASS) para el período 2009-2011. Estimaron que el 1\% de las personas de mayores ingresos se apropió de entre el 12 y el 14\% del ingreso total, situación estable en el período; observaron que la ECH subestima la participación del 1\% de las personas de mayores ingresos aproximadamente en 3\%, y concluyeron: «Si bien la captación de los ingresos altos en esta encuesta es razonable, resulta preocupante el hecho de que, en los tramos muy altos de la distribución, las brechas de ingresos entre ambos registros tiendan a acrecentarse en el corto período estudiado» (p. 120). De Rosa y Vigorito concluyeron, tras haber utilizado también información tributaria: «Los perceptores de altos ingresos se ubican principalmente en empresas de mayor volumen de ventas, en sociedades anónimas y en empresas de más de 20 empleados» (2015, p. 31). De Rosa, Siniscalchi, Vigorito y Willebald (2016) ratificaron las conclusiones de la literatura que corrige la información de la ECH con datos de recaudación de la DGI.

Estos trabajos no tuvieron en cuenta: i) las exoneraciones, es decir, ingresos que no están gravados y por lo tanto no generan recaudación; ii) los ingresos generados en el país y transferidos al exterior por intereses de inversiones en cartera (IEC) o utilidades de inversiones extranjeras directas (IED); iii) las utilidades reinvertidas que no pagan IRPF pero sus propietarios son personas físicas; iv) la evasión y la elusión.

e. Como antecedentes recientes de la búsqueda de un camino alternativo a los enfoques de distribución funcional y personal, con un enfoque de economía política contemporánea que mantiene la correspondencia entre las categorías de ingresos y la estructura social pero no se limita a tres clases sociales, corresponde destacar tres trabajos. Giometti (2015) estimó la participación de las clases populares en el PIB entre 2001 y 2013, considerándola integrada por los distintos tipos de asalariados no directivos, por los trabajadores por cuenta propia sin local o inversión, los trabajadores de los hogares no remunerados, los cooperativistas, los miembros de programas sociales de empleo y todos los desocupados, además de los jubilados y pensionistas que perciben hasta cierto umbral de ingresos. Notaro (2016) estimó los ingresos del capital utilizando información tributaria. Oyantçabal y Sanguinetti (2017) analizaron las actividades agropecuarias y destacaron que se observan cuatro formas principales de ingresos percibidos por terratenientes, capitalistas, asalariados y productores mercantiles directos. 
f. Desde 1999 casi todas las investigaciones sobre distribución del ingreso tienen como objeto la distribución personal o familiar; las tres excepciones que investigaron con un enfoque de economía política se limitaron a los ingresos de los sectores populares, del capital o a la actividad agropecuaria. La pregunta inicial del marco conceptual no ha sido contestada y su respuesta requiere otro enfoque, otras fuentes y otros procedimientos para la construcción de indicadores.

\section{Resultados para Uruguay}

Se construyeron dos indicadores: la importancia relativa de los grupos sociales en la captación de ingresos que forman parte del PIB pm (cuadro 1) y la relación entre los ingresos del capital y los de los obreros y empleados (cuadro 2). Los resultados se presentan en el cuadro 1 como porcentajes, y las últimas dos filas permiten calcular las magnitudes en pesos corrientes y en dólares.

El total de ingresos del capital fluctúa con un máximo del 32,5\% en 2010 y un descenso hasta el $28,1 \%$ en 2014 . Teniendo en cuenta que el PIB pm crece durante todo el período, estas oscilaciones se reflejan en un aumento de la magnitud de los ingresos del capital, que en los tres últimos años del período se estiman en un promedio anual de 16.100 millones de dólares. Sin considerar la depreciación, los ingresos del capital extranjero descienden como resultado de una reducción del $1 \%$ del pago de intereses de deuda externa. Los ingresos del capital nacional presentan un máximo del 23,6\% en 2010 para descender luego hasta un 20,2\% en 2014; el principal descenso se observa en las utilidades, dividendos, intereses y rentas recaudados por la DGI o exonerados, con un máximo del 18,1\% en 2012 hasta 15,6\% en 2014 (cuadro A.1). Del total de ingresos del capital nacional registrados por la DGI como gravados y como exonerados después del pago de impuestos, en el período 2010-2014 solo el 7,8\% fue captado por la ECH del INE (Notaro, 2016). 
Cuadro 1. Distribución social del ingreso, 2008-2014, como porcentaje del PIB pm, en millones de pesos corrientes

\begin{tabular}{|c|c|c|c|c|c|c|c|}
\hline & 2008 & 2009 & 2010 & 2011 & 2012 & 2013 & 2014 \\
\hline 1. Capital & 27,8 & 29,4 & 32,5 & 29,8 & 30,3 & 29 & 28,1 \\
\hline 1.1. Extranjero & 5,5 & 5 & 4,9 & 4,4 & 3,7 & 3,7 & 4 \\
\hline 1.2. Nacional & 18,3 & 20,4 & 23,6 & 21,3 & 22,6 & 21,3 & 20,2 \\
\hline 1.3. Depreciación & 4 & 4 & 4 & 4 & 4 & 4 & 4 \\
\hline 2. Salarios (efectivo) & 21,8 & 21,8 & 20,8 & 22,6 & 22,6 & 22,2 & 23,2 \\
\hline 2.1. Obreros y empleados & 17,6 & 17,2 & 16,4 & 17,2 & 17,6 & 17,2 & 18,1 \\
\hline 2.2 Otros & 4,2 & 4,7 & 4,4 & 5,4 & 5,1 & 4,9 & 5,2 \\
\hline 3. Ingresos mixtos & 6,5 & 6 & 5,7 & 5,7 & 5,1 & 5,1 & 5,2 \\
\hline 3.1. Cuenta propia s/l & 0,4 & 0,4 & 0,3 & 0,3 & 0,3 & 0,2 & 0,2 \\
\hline 3.2. Cuenta propia $\mathrm{c} / \mathrm{l}$ & 6,1 & 5,7 & 5,4 & 5,4 & 4,8 & 4,9 & 5 \\
\hline $\begin{array}{l}\text { 4. Pasividades y transferen- } \\
\text { cias }\end{array}$ & 10,5 & 10,8 & 10,5 & 10,2 & 10,3 & 10,4 & 10,2 \\
\hline 4.1 Pasividades & 8,8 & 9 & 9 & 8,4 & 8,6 & 8,7 & 8,4 \\
\hline 4.2 Transferencias públicas & 1,7 & 1,8 & 1,5 & 1,7 & 1,7 & 1,7 & 1,9 \\
\hline 5. Estado & 13,6 & 13,3 & 15,2 & 14,8 & 14,1 & 15,3 & 14 \\
\hline 6. Ingresos varios & 21,8 & 21,2 & 16,9 & 18,3 & 18,1 & 18,9 & 19,9 \\
\hline 7. PIB pm & 100 & 100 & 100 & 100 & 100 & 100 & 100 \\
\hline 8. PIB pm millones de $\$$ & $636.150,9$ & $714.523,4$ & $808.078,5$ & $926.356,1$ & $1.041 .210,5$ & $1.178 .331,7$ & $1.330 .508,4$ \\
\hline 9. PIB pm millones de USD & $30.366,1$ & $31.660,9$ & $40.284,5$ & $47.962,4$ & $51.265,0$ & $57.531,0$ & $57.236,0$ \\
\hline
\end{tabular}

Fuentes: ECH del INE, SCN y BP del BCU, DGI y BPS.

En la masa salarial se diferencian los salarios de obreros y empleados, que fluctuaron levemente en torno al $17 \%$ del PIB pm, con leve ascenso en los últimos tres años del período; la magnitud promedio anual entre 2012 y 2014 fue de 9.759,2 millones de dólares. En los salarios de otros, el componente más importante son los profesionales universitarios asalariados, cuyos ingresos fluctuaron entre $4 \%$ y $5 \%$. Los correspondientes a las Fuerzas Armadas y el personal político son de baja magnitud (cuadro A.2).

Los ingresos de los trabajadores por cuenta propia de la ECH, que son iguales a los ingresos mixtos en el SCN, presentan una ligera tendencia al descenso desde el 6,5\% hasta 
el 5,2\% por la reducción de los ingresos de los trabajadores por cuenta propia con local, dado que los sin local son poco relevantes.

Se incluyen las pasividades y las transferencias públicas que registra la ECH. Las primeras fluctúan entre $8,4 \%$ y $9 \%$, con un promedio anual durante los últimos tres años del período de 4.740 .6 millones de dólares. En las transferencias públicas, que fluctúan entre el 1,5\% y el 1,7\%, se incluyen los pagos por seguros de desempleo y de salud, así como los diversos tipos que se realizan a los hogares de menores ingresos.

En los ingresos del Estado se agregan a los impuestos indirectos menos subsidios que forman parte del PIB pm los impuestos directos recaudados por la DGI y los aportes al BPS tanto personales como patronales, teniendo en cuenta que los ingresos de los grupos sociales se registraron luego del pago de estos impuestos y contribuciones. En el período presentan un leve aumento, desde algo más del $13 \%$ al principio del período hasta algo más del 14\% en los últimos años. El principal componente son los impuestos indirectos menos subsidios, con algo más del $12 \%$, seguidos por las contribuciones a la seguridad social, que desde algo más del 5\% aumentan hasta algo más del 6\% a fin del período (cuadro A.3).

Se estima también un grupo de ingresos varios que no son percibidos por ninguno de los grupos sociales identificados. Incluyen las contribuciones a las cajas paraestatales, las utilidades de las empresas que pagan el impuesto a la enajenación de bienes agropecuarios (IMEBA) y no pagan IRAE, así como una estimación de la evasión de IRPF (cuadro A.4); los dos últimos podrían incluirse como ingresos del capital. De este grupo, algo más del $13 \%$ del PIB pm son ingresos con perceptores no identificados, lo que resulta de tomar en todas las estimaciones anteriores hipótesis de mínima, de modo que los ingresos resultantes fueran un piso confirmado. Los ingresos con perceptores no identificados podrían corresponder a una mayor magnitud de la depreciación, teniendo en cuenta que la inversión creció en el período, hubo mayor evasión de los impuestos sobre los ingresos del capital, mayores utilidades de los productores agropecuarios que pagan IMEBA, así como subestimaciones en la ECH de los salarios y de los ingresos de los trabajadores por cuenta propia.

En el cuadro 2 se presenta el cociente entre los ingresos del capital (cuadro 1, fila 1, Capital) y los ingresos del trabajo, con tres niveles de agregación. En primer lugar, con los ingresos de obreros y empleados (cuadro 1, fila 2.1), un grupo social homogéneo de asalariados, se observa que los primeros fueron casi el doble de los segundos en $2010 \mathrm{y}$ luego descendieron hasta ubicarse en una vez y media en 2014. Segundo, con relación al total de salarios del que se deduce la imputación por la cobertura de salud que realiza el INE en la ECH, los ingresos del capital llegaron a un máximo de vez y media el total de salarios en 2010, para descender luego hasta ubicarse en un $21,2 \%$ por encima. Tercero, con relación al total de ingresos del trabajo sumando los salarios y los ingresos de los 
trabajadores por cuenta propia, la evolución fue la misma que en los dos anteriores, con diferencias de menor magnitud.

Cuadro 2. Ingresos relativos del capital, 2008-2014

\begin{tabular}{|c|c|c|c|c|c|c|c|}
\hline & $\begin{array}{l}200 \\
8\end{array}$ & $\begin{array}{l}200 \\
9\end{array}$ & $\begin{array}{l}201 \\
0\end{array}$ & $\begin{array}{l}201 \\
1\end{array}$ & $\begin{array}{l}201 \\
2\end{array}$ & $\begin{array}{l}201 \\
3\end{array}$ & $\begin{array}{l}201 \\
4\end{array}$ \\
\hline $\begin{array}{l}\text { 2.1. Obreros y emplea- } \\
\text { dos }\end{array}$ & 157,7 & 171,2 & 197,4 & 173 & 172,4 & 168,5 & 155,8 \\
\hline 2. Salarios (efectivo) & 127,2 & 134,6 & 155,7 & 131,8 & 133,7 & 131 & 121,2 \\
\hline $2+3$. Ingresos del trabajo & 98 & 105,4 & 122,3 & 105,3 & 109 & 106,5 & 98,9 \\
\hline
\end{tabular}

Fuente: Cuadro 1.

\section{Conclusiones finales}

La originalidad del presente artículo con relación a la literatura sobre la distribución del ingreso en el Uruguay se manifiesta en cuatro aspectos: la estimación de la distribución del total de ingresos generados entre grupos sociales, la identificación de los ingresos del capital con información tributaria que permitió superar la insuficiente captación de la ECH del INE, la inclusión de los ingresos del capital extranjero que la ECH no puede captar, y la desagregación de la masa salarial identificando los ingresos de obreros y empleados. Cabe observar que, a diferencia del SCN, los ingresos se registran después del pago de impuestos y aportes a la seguridad social.

La metodología utilizada permitió superar las críticas a la economía política clásica, recuperar la relación de los ingresos con los grupos que integran la estructura social y retomar las preocupaciones de las investigaciones previas a la dictadura. Las limitaciones del enfoque original de la economía política para analizar la distribución del ingreso a principios del siglo XXI en los países de América Latina pueden ser superadas $\mathrm{y}$, por lo tanto, no llevan necesariamente a descartarlo y limitar las investigaciones a la distribución personal, familiar o funcional.

Las estimaciones realizadas de la distribución social del ingreso en Uruguay en el período analizado permiten concluir que en la relación de los ingresos de los grupos en el PIB pm predominó la estabilidad con ligeras fluctuaciones. En la relación de los ingresos del capital con los de los obreros y empleados se aprecia una importante reducción.

Se calcularon los promedios anuales de los últimos tres años del período, considerando que el resultado es más representativo que las leves variaciones. Los ingresos del 
capital se ubicaron en un $29,1 \%$ del PIB pm, los de los obreros y empleados en un $17,6 \%$, los de los pasivos en un 8,6\% y los de los trabajadores por cuenta propia en un 5,1\%.

Las primeras investigaciones sobre distribución del ingreso en el Uruguay se debatieron contra la insuficiencia de información. Hasta 1980 la EH solo relevaba los ingresos del trabajo y hasta 2006 la cobertura era únicamente urbana. Las investigaciones iniciales comenzaban definiendo el problema a investigar, evaluaban las fuentes, diseñaban procedimientos de estimación buscando identificar los ingresos de los diversos grupos socioeconómicos que formaban parte de la estructura social del país y consideraban relevante conceptualizar y estimar los ingresos del capital.

En el período posterior a la dictadura hasta la actualidad se observa en casi todas las investigaciones un cambio en la forma de abordar el tema, en los marcos teóricos, en las metodologías y en los procedimientos de construcción de indicadores. El punto de partida fueron las técnicas de medida diseñadas en el hemisferio norte para analizar la distribución del ingreso entre hogares y personas, que abandonaron la evaluación de las fuentes, el diseño de procedimientos de estimación originales, el análisis de las relaciones entre los ingresos y los grupos sociales. Los trabajos que investigaron la distribución funcional a principios del siglo XXI señalaron como marco teórico a los economistas clásicos, como Smith y Ricardo, lo que es equivocado porque estos analizaron la distribución del ingreso entre clases sociales y no entre factores productivos.

En los últimos años del siglo xx los investigadores destacaron la subestimación de los altos ingresos en las EH y a principios del siglo XXI realizaron algunos intentos de superarla con información tributaria. Las investigaciones que aceptan sin relativizar los ingresos de capital registrados por la ECH y con esos datos calculan índices de Gini subestiman la concentración del ingreso por tres razones: a) no tienen en cuenta las utilidades reinvertidas, b) tampoco las transferencias al exterior de ingresos generados en el país, y c) como se ha comprobado, la ECH solo capta una parte menor de los ingresos del capital. Estos trabajos incurren en lo que se puede definir como the household surveys trap.

El mejor registro de los ingresos del capital aumentaría los correspondientes a los deciles superiores, dado que para percibirlos se requiere tener propiedad y como precondición tener capacidad de ahorro. Es una hipótesis convincente considerar que las familias y las personas que tienen capacidad de ahorro pertenecen a los tres deciles de mayores ingresos y, por el contrario, en los tres deciles de menores ingresos no hay capacidad de ahorro.

Se observa una ausencia y dos paradojas: la ausencia es la estimación de los ingresos del capital, categoría que no se utiliza por la corriente predominante en el medio académico uruguayo ni en las estadísticas oficiales. La primera paradoja es que los hogares uruguayos declaren más ingresos del capital a la DGI que a la ECH, como en la mayoría de los países latinoamericanos, y la segunda, que se define como la paradoja de 
Gini, es que cuando se venden activos a no residentes se reducen los ingresos del capital disponibles en el país que podría registrar la ECH y, como consecuencia, mejora la distribución del ingreso de acuerdo este índice. En países en los que la propiedad del capital y de la tierra de no residentes es irrelevante, esta paradoja es igualmente irrelevante. Pero en AL las IED, los recursos naturales y los activos financieros propiedad de no residentes son importantes y aumentaron aceleradamente durante los primeros años del siglo XXI, con lo que la paradoja adquiere mayor importancia.

El índice de Gini, como todos los indicadores que se construyen para medir categorías, tiene condicionantes ideológicas derivadas de la teoría de la que forman parte y también históricas, derivadas de las particularidades del momento y del lugar. Su cálculo con información de las EH en Uruguay es científicamente equivocado y políticamente conservador; la subestimación de los ingresos del capital se utiliza como fundamento científico por el sistema político para medir su capacidad contributiva, lo que desestimula medidas redistributivas como los impuestos que los gravan.

Las limitaciones de la ECH para registrar los altos ingresos se reconocieron desde hace más de 25 años y, sin embargo, no se adoptaron medidas para superarlas. Se pueden señalar múltiples responsabilidades. En primer lugar, del INE, que difunde los resultados de la ECH sin contrastarlos con otras fuentes, operación que le permitiría apreciar que los ingresos del capital están subregistrados y debería corregirlos, o por lo menos advertir a los usuarios. En segundo lugar, del BCU, que elabora el SCN, interrumpió la información sobre la cuenta de ingresos en 2005 y perdió el interés en los ingresos del capital, que se manifestaban en la construcción del SCN en 1965. La decisión del directorio del BCu de suspender la construcción de la cuenta ingresos resulta incomprensible considerando que cuenta con un numeroso y calificado equipo técnico, que dispone de la información de la DGI y que construye indicadores de los más diversos aspectos de la actividad económica. Tercero, de los investigadores que utilizan la información de la ECH en estimaciones sobre la distribución del ingreso, sin destacar las limitaciones ni exigir al INE que mejore el registro de los ingresos del capital. Los trabajos que intentaron superar las limitaciones de captación de la ECH utilizando información tributaria sobre los altos ingresos familiares y personales sin incluir la totalidad de los ingresos del capital legitimaron, con un fundamento aparentemente científico, un grado de concentración del ingreso menor que el real.

Los fundadores de la economía política no disponían de los actuales instrumentos de medida y tampoco de la informática que los potenció desde fines del siglo XX. No tuvieron otra opción, ante la imposibilidad de medir con precisión, que observar el contexto histórico y reflexionar con rigor. 


\section{Anexo metodológico}

Se explicitan las fuentes de información y los procedimientos utilizados para la construcción de indicadores. En todos los casos en que se definen hipótesis para la estimación se toma como criterio fijar un mínimo de ingresos comprobados.

El período está delimitado por la disponibilidad de información; 2008 es el primer año con la información completa de recaudación de impuestos sobre los ingresos del capital y 2014 es el último, hasta este momento, con el monto de los ingresos exonerados de dichos impuestos. El total de ingresos medido por el PIB pm a precios corrientes se toma del SCN construido por el BCU.

Se procesaron los microdatos de la ECH del INE, lo que permitió estimar los ingresos del trabajo de la ocupación principal y secundaria por categoría ocupacional (co) y tipo de ocupación (TO). De acuerdo a la legislación vigente, los empleadores operan como agentes de retención, de modo que los hogares reciben los ingresos deducidos los impuestos directos y los aportes a la seguridad social. No se incluyen los ingresos imputados en la ECH por concepto de valor locativo ni por cuota mutual porque no forman parte del PIB pm.

Los ingresos del capital incluyen en primer lugar los gravados y los exonerados por la DGI, después del pago de impuestos. La reforma tributaria que entró en vigencia en 2007 grava todas las formas de ingresos del capital. Teniendo en cuenta las tasas de los diversos impuestos es posible calcular los ingresos del capital sumando los montos gravados y exonerados, incluyendo las utilidades de las empresas, los intereses de todo tipo, como de depósitos bancarios o de deuda pública, y los alquileres (de tierras, viviendas, locales comerciales, maquinarias, vehículos, etc.) (cuadro A.1, 2.1, Ut. Div. Int. y R.). También Medeiros, Ferreira de Souza y Ávila de Castro (2015) incluyeron estos ingresos estimados con información tributaria, señalando que los de las personas jurídicas pertenecen a personas físicas. Se estima una magnitud comprobada que representa un piso o un mínimo (Notaro, 2016). Se consideraron también ingresos del capital los salarios de los directivos y gerentes de empresas privadas (T01, c0 asalariado privado), así como los ingresos que los patrones declaran como resultado del trabajo (Co patrón, cuadro A.1, 2.2, Rem. patrones), que son inseparables de la propiedad del capital (Glyn, 2009; Wulff Gobetti y Orair, 2017). Se sumó también la evasión en el pago del impuesto a la renta de las actividades económicas (IRAE) estimada por la DGI (Dirección General de Impositiva [DGI], 2013). Por último, se agregó una estimación de la depreciación del capital como se consideró en la primera versión de las Cuentas Nacionales (BRoU, 1965, p. A 64-A 65); entre 1955 y 1967 representó el 3,8\% del PIB pm (Banco Central del Uruguay [BCU], 1969). Amarante y Carella (2000) estimaron la depreciación como un 3,9\% del PIB en el período 1989-1995 y, teniendo en cuenta el importante aumento de la inversión privada en los últimos años, tomar un porcentaje del 4\% del PIB 
se puede considerar un piso (cuadro A.1). El BCU solo estima las rentas de la propiedad en la cuenta del resto del mundo (RM), es decir, las que resultan de las relaciones con no residentes, que comprenden los dividendos y las utilidades de la inversión extranjera directa (IED), así como los intereses de todas las operaciones. Cuando estos ingresos son reinvertidos total o parcialmente en el Uruguay, en la balanza de pagos (BP) se registra un egreso por el total de ingresos del capital extranjero y un ingreso por las cantidades reinvertidas. No son captados por las EH porque corresponden a hogares que no residen en el país.

Cuadro A.1. Ingresos del capital como porcentaje del PIB pm

\begin{tabular}{|l|r|r|r|r|r|r|r|}
\hline \multicolumn{1}{|c|}{} & 2008 & 2009 & 2010 & 2011 & 2012 & 2013 & 2014 \\
\hline Total & 27,8 & 29,4 & 32,5 & 29,8 & 30,3 & 29 & 28,1 \\
\hline 1. Extranjero & 5,5 & 5 & 4,9 & 4,4 & 3,7 & 3,7 & 4 \\
\hline 1.1 Utilidades & 2,7 & 2,4 & 2,8 & 2,6 & 2,3 & 2,1 & 2,4 \\
\hline 1.2 Intereses & 2,8 & 2,6 & 2,1 & 1,8 & 1,4 & 1,6 & 1,6 \\
\hline 2. Nacional & 18,3 & 20,4 & 23,6 & 21,3 & 22,6 & 21,3 & 20,2 \\
\hline 2.1 Ut. Div. Int. y R. & 12 & 13,8 & 17,7 & 15,8 & 18,1 & 16,3 & 15,6 \\
\hline 2.2 Rem. patrones & 4,5 & 4,4 & 3,9 & 3,7 & 2,7 & 3 & 2,9 \\
\hline $\begin{array}{l}\text { 2.3 Directores y gerentes priva- } \\
\text { dos }\end{array}$ & 1 & 1,2 & 0,9 & 0,8 & 0,7 & 0,8 & 0,7 \\
\hline 2.4 Evasión irae & 0,8 & 1 & 1,2 & 1 & 1 & 1,2 & 1 \\
\hline 3. Depreciación & 4 & 4 & 4 & 4 & 4 & 4 & 4 \\
\hline
\end{tabular}

Fuente: DGI, ECH del INE y SCN del BCU.

Los salarios (cuadro A.2) se desagregan de acuerdo a los To que consideran grupos de tareas similares (International Labour Office [ILO], 1988). En el ingreso total de las co de asalariados privados y asalariados públicos se diferencian los salarios de obreros y empleados, y los de los de otros, que incluye las Fuerzas Armadas (To0), el personal político (т01) y los profesionales universitarios (т02). También Portes y Hoffman (2003) separaron los salarios de ejecutivos y profesionales universitarios. 
Cuadro A.2. Salarios (sin cuota mutual) como porcentaje del PIB pm

\begin{tabular}{|l|l|l|l|l|l|l|l|}
\cline { 2 - 8 } \multicolumn{1}{c|}{} & 2008 & 2009 & 2010 & 2011 & 2012 & 2013 & 2014 \\
\hline Total & 21,8 & 21,8 & 20,8 & 22,6 & 22,6 & 22,2 & 23,2 \\
\hline 1. Obreros y empleados & 17,6 & 17,2 & 16,4 & 17,2 & 17,6 & 17,2 & 18,1 \\
\hline 2. Profesionales & 3,9 & 4,3 & 4 & 5,1 & 4,6 & 4,4 & 4,7 \\
\hline 3. Fuerzas armadas & 0,1 & 0,2 & 0,2 & 0,2 & 0,2 & 0,2 & 0,2 \\
\hline $\begin{array}{l}\text { 4. Directores y gerentes públi- } \\
\text { cos }\end{array}$ & 0,2 & 0,2 & 0,2 & 0,2 & 0,3 & 0,3 & 0,3 \\
\hline
\end{tabular}

Fuente: ECH del INE y SCN del BCU.

Los ingresos del trabajo aplicado sobre medios de producción del trabajador se toman de las ECH, que los consideran ingresos de la co cuenta propia. Coinciden con la definición de ingresos mixtos del SCN, que son los que perciben los «trabajadores no asalariados o autónomos», considerados personas que trabajan para sí mismas (Banco Central del Uruguay [BСU], 2009, p. 121).

Los ingresos del Estado que se registran en el PIB pm son, en primer lugar, los impuestos indirectos menos subvenciones, sobre los productos y sobre la producción que estima el BCU. En el cálculo del SCN los ingresos de los trabajadores asalariados y de los trabajadores por cuenta propia se toman antes del pago de impuestos y aportes; como la ECH los deduce, se incluyen como ingresos del Estado. Los aportes a la seguridad social sobre salarios, de los trabajadores y de los patrones, se toman de los ingresos del BPS por este concepto (no todos los ingresos); están incluidos los ingresos que se transfieren a las administradoras de fondos de ahorro previsional (AFAP). Por último, los ingresos por concepto de pasividades y transferencias públicas que se incluyen en el cuadro 1 se deducen del total de ingresos del Estado para evitar duplicaciones (cuadro A.3). 
Cuadro A.3. Ingresos del Estado como porcentaje del PIB pm, 2008-2014

\begin{tabular}{|l|r|r|r|r|r|r|r|}
\hline & 2008 & 2009 & 2010 & 2011 & 2012 & 2013 & 2014 \\
\hline Total & 13,6 & 13,3 & 15,2 & 14,8 & 14,1 & 15,3 & 14 \\
\hline $\begin{array}{l}\text { 1. Impuestos indirectos - Subsid- } \\
\text { ios }\end{array}$ & 13 & 12,2 & 12,6 & 12,7 & 12,4 & 12,3 & 12,2 \\
\hline 2. irpf & 2,2 & 2 & 2,1 & 2,3 & 2,5 & 2,6 & 2,7 \\
\hline 2.1. Categoría 1 & 0,3 & 0,3 & 0,3 & 0,3 & 0,4 & 0,4 & 0,4 \\
\hline 2.2 Categoría 2 & 1,9 & 1,7 & 1,8 & 2 & 2,2 & 2,2 & 2,4 \\
\hline 3. irae & 2,5 & 2,5 & 2,6 & 2,3 & 2,3 & 2,7 & 2,2 \\
\hline 4. iass & 0,1 & 0,3 & 0,3 & 0,3 & 0,4 & 0,4 & 0,4 \\
\hline 5. irnr & 0,2 & 0,2 & 0,2 & 0,3 & 0,2 & 0,2 & 0,2 \\
\hline 6. bps & 5,2 & 5,4 & 5,6 & 5,9 & 5,9 & 6 & 6,3 \\
\hline 7. Empresas públicas & 0,9 & 1,4 & 2,2 & 1,1 & 0,8 & 1,5 & 0,2 \\
\hline 8. Pasividades y transferencias & $-10,5$ & $-10,8$ & $-10,5$ & $-10,2$ & $-10,3$ & $-10,4$ & $-10,2$ \\
\hline
\end{tabular}

Fuente: BCU, DGI, BPS y Ministerio de Economía y Finanzas (MEF).

En los ingresos varios se establece una hipótesis moderada de evasión de IRPF de un $15 \%$, teniendo en cuenta los porcentajes de evasión en AL, las estimaciones de Grosskopff (1991), de Fuentes y Mendive (1996) y la estimación de algo más de 500 millones de dólares anuales en el pago de impuestos sobre arrendamientos (Notaro, 2016) (cuadro A.4). El porcentaje en el PIB pm de los aportes a las cajas Militar, Policial, Bancaria, Profesional y Notarial se toman de Amarante y Carella (2000). Por último, como diferencia entre los ingresos identificados y el PIB pm se delimita un porcentaje de ingresos de perceptores no identificados en torno al 14\%, que es resultado de las hipótesis de mínimos en las estimaciones de los demás ingresos. 
Cuadro A.4. Ingresos varios como porcentaje del PIB pm

\begin{tabular}{|l|l|l|l|l|l|l|l|}
\cline { 2 - 7 } \multicolumn{1}{c|}{} & 2008 & 2009 & 2010 & 2011 & 2012 & 2013 & 2014 \\
\hline Total & 21,8 & 21,2 & 16,9 & 18,3 & 18,1 & 18,9 & 19,9 \\
\hline 1. Cajas paraestatales & 3,5 & 3,5 & 3,5 & 3,5 & 3,5 & 3,5 & 3,5 \\
\hline $\begin{array}{l}\text { 2. Utilidades en } \\
\text { imeba }\end{array}$ & 0,3 & 0,2 & 0,2 & 0,2 & 0,2 & 0,2 & 0,2 \\
\hline 3. Evasión irpf & 2,1 & 2,3 & 0,7 & 1,1 & 0,7 & 1,4 & 1,5 \\
\hline 4. Indeterminados & 16 & 15,1 & 12,5 & 13,5 & 13,7 & 13,8 & 14,7 \\
\hline
\end{tabular}

Fuente: SCN y BP del BCU, DGI.

\section{Referencias bibliográficas}

AlVAREDO, F. y GASPARINI, L. (2013). Recent trends in inequality and poverty in developing countries. La Plata: CEDLAS. (Documento de Trabajo, 15). Recuperado de http://hdl.handle.net/10419/127675.

Amarante, V. y Carella, A. (2000). «Distribución del ingreso: ajuste a las estimaciones tradicionales y una propuesta alternativa». Quantum, 5(11), 16-37.

AmARANTE, V. Y JimÉNEZ, J. P. (2015). «Desigualdad, concentración y rentas altas en América Latina». En J. P. JimÉNEZ (ed.), Desigualdad, concentración del ingreso y tributación sobre las altas rentas en América Latina. Santiago de Chile: CEPAL.

AMARANTE, V. Y Vigorito, A. (2011). «Los futuros posibles de la desigualdad de ingresos». En R. ARocena y G. CAETANo (coords.), La aventura uruguaya: ¿Naides más que naides? (pp. 23-47). Montevideo: Random House Mondadori.

ARAAR, A (2008). Social classes, inequality and redistributive policies in Canada. Quebec: CIRPÉE.

ARDEnte, A., Rossi, T. y DíAz, F. (2003). Crecimiento y distribución del ingreso en el Uruguay del siglo XX. (Tesis de Licenciatura en Economía). Facultad de Ciencias Económicas y Administración, Montevideo.

ATKInSON, A. B. (1989). The economics of inequality. Oxford: Oxford University Press.

ATKINSON, A. B. (2007). «Measuring top incomes: Methodological issues». En A. B. ATKINSON y T. PIKETTY (eds.), Top incomes over the Twentieth Century: A contrast between continental european and english-speaking countries. Oxford: Oxford University Press. 
Atkinson, A. B. y Bourguignon, F. (eds.) (2000). Handbook of income distribution, Ámsterdam: Elsevier.

AtKinson, A. B. y Bourguignon F. (eds.) (2015). Handbook of income distribution: Volumen 2A-2B. Ámsterdam: Elsevier.

BCU (1969). Cuentas nacionales. Montevideo: BCU.

BCU (1980). Tasas de retorno al capital. Montevideo: BCU.

BCU (2009). Revisión integral de las Cuentas Nacionales 1997-2008: Metodología. Montevideo: BCU.

BROU (1965). Cuentas nacionales. Montevideo: BROU.

BARRETT, Ch. y ESTEBAN, J. (2005). «Social groups and economic inequality». Journal of Economic Inequality, 3(3), 187-191.

BENSIÓN, A. y CAUMONT, J. (1979). Política económica y distribución del ingreso en el Uruguay: 1970-1976. Montevideo: Acali.

BÉRTOLA, L. (2001). Income distribution and the Kuznets curve: Argentina and Uruguay since the 1870. Montevideo: FCS.

Bértola, L. (2005). «A 50 años de la curva de Kuznets: Crecimiento económico y distribución del ingreso en Uruguay y otras economías de nuevo asentamiento desde 1870». Investigaciones de Historia Económica, 1(3), 135-176.

BisognO, M. y GRAU, C. (1989). «La tributación indirecta y la distribución del ingreso». Suma, 4(6), 17-45.

BLANDEN, J.; GREGG, P. y MACMILLAN, L. (2013). Intergenerational persistence in income and social class: The impact of within-group inequality. Londres: CEP.

Bucheli, M. y Rossi, M. (1994). Distribución del ingreso en el Uruguay: 1986-1992. Montevideo: DECON.

BuCHELI, M. y FurTADO, M. (2000). La contribución de las distintas fuentes de ingreso a la evolución de la desigualdad en el Uruguay urbano: 1986-1997. Montevideo: CEPAL.

Bucheli, M. y FurTADo, M. (2001). La distribución del ingreso en Uruguay 1986-1999: Alternativas para su medición. Montevideo: CEPAL.

Bucheli, M. y FuRTADO, M. (2004). Uruguay 1998-2000: La distribución del ingreso en la crisis. Santiago de Chile: CEPAL.

BuCHELI, M. y GonzÁLEZ, C. (2010). «La desigualdad de los ingresos en Uruguay». En E. ACCINELli y O. SALAS (eds), Crecimiento y distribución del ingreso en América Latina (pp. 335-363). México: FCS.

BuRdín, G.; ESPONDA, F. y VIGORITO, A. (2015). «Desigualdad y altas rentas en el Uruguay: Un análisis basado en los registros tributarios y las encuestas de hogares del período 2009-2011». En J. P. JiMÉNEZ (ed.), Desigualdad, concentración del ingreso y tributación sobre las altas rentas en América Latina. Santiago de Chile: CEPAL. 
Burdin, G.; De RoSA, M. y A. Vigorito (2015). Sectores de altos ingresos en Uruguay: participación relativa y patrones de movilidad en el período 2009-2012. Montevideo: IECON. (Serie Documentos de Trabajo, 03).

CHAI, A. y KaUS, W. (2012). Signalling to whom? Conspicuous spending and the local density of the social group income distributionk. Jena: Evolutionary Economic Group. (Papers on Economics and Evolution, 1218).

CIDE (1966). Plan Nacional de Desarrollo Económico y Social 1965-1974: Tomo I. Desarrollo de la economía en su conjunto. Montevideo: CIDE.

CEPAL (1989). Estructura socio-ocupacional y distribución del ingreso en el Uruguay (1984-1988). Montevideo: CEPAL.

CoRNIA, G. A. (2014). Income inequality in Latin America: Recent decline and prospects for its further reduction. Santiago de Chile: ECLAC. (Macroeconomics of Development Series, 149).

CoRRAZO, R. y SANDER, N. (2005). La distribución del ingreso en Uruguay: 1960-2000. (Tesis de Licenciatura en Economía). Facultad de Ciencias Económicas y Administración, Montevideo.

CURTIS, J. y ANDERSEN, R. (2015). «How social class shapes attitudes on economic inequality: The competing forces of self-interest and legitimation». International Review of Social Research, 5(1): 4-19.

De Ferranti, D.; Perry, G. E.; Ferreira, F. H. G.; Walton, M.; Coady, D.; Cunningham, W. ... Wodon, Q. (2003). Inequality in Latin America and the Caribbean: Breaking with History? Washington: The World Bank.

DE RosA, M. (2017). «Desigualdad en Uruguay: entre la evidencia y el relato». Hemisferio Izquierdo, 13.

De Rosa, M.; Siniscalchi, S.; Vigorito, A. y Willebald, H. (2016). El estado del arte de los estudios distributivos en Uruguay. Montevideo: IECON.

DE RosA, M. y Vigorito, A. (2015). Los vínculos entre la distribución personal y funcional del ingreso: Una exploración para Uruguay en base a registros tributarios. Montevideo: AECID.

DGI (2013). Estimación de la evasión en el impuesto al valor agregado mediante el método del consumo 2000-2012. Montevideo: DGI.

Dовв, M. (1988). Teorías del valor y de la distribución desde Adam Smith: Ideología y teoría económica. México, Siglo XXI.

FÉLIZ, M.; FERNÁNDEZ, L. y LÓPEZ, E. (octubre 2010). «Estructura de clase, distribución del ingreso y políticas públicas». Ponencia presentada a las 3. as Jornadas de Economía Crítica, Rosario, Argentina.

Fuentes, Á. y Mendive, C. (1996). Evaluación de la captación del ingreso de los hogares. Montevideo: CEPAL. 
GasParini, L.; Cicowiez, M. y Sosa Escudero, W. (2014). Pobreza y desigualdad en America Latina: Conceptos, herramientas y aplicaciones. La Plata: CEDLAS. (Documentos de Trabajo, 171).

GiometTI, B. (abril 2015). «Evolución de los ingresos de los sectores populares entre 2001 y 2013». Ponencia presentada a las Jornadas del Espacio de Economía Crítica, Montevideo.

GLYN, A. (2009). «Functional distribution and Inequality». En W. SALVERDA, B. NOLAN y T. SMEEDING (eds.), The Oxford handbook of economic inequality. Oxford: Oxford University Press. (Oxford Handbooks in Economics).

Gómez SABAínI, J. C. y Rossignolo, D. (2015). «La tributación sobre las altas rentas en América Latina». En J. P. JiMÉNEZ (ed.), Desigualdad, concentración del ingreso y tributación sobre las altas rentas en América Latina. Santiago de Chile: CEPAL.

Gradin, C. y Rossi, M. (2001). The distribution of income in Uruguay: The effects of economic and institutional reforms. Montevideo: FCS.

GraU, C. y Lagomarsino, G. (2002). Estructura tributaria de Uruguay: Su impacto en la distribución del ingreso de los hogares. Montevideo: FCU.

GROSSKOFF, R. (1991). Análisis y ajuste de los ingresos investigados en las encuestas de hogares. Montevideo: FCEA.

HARBERGER, A. y WiSECARVER, J. (1978). Tasas de retorno al capital en los ámbitos privado y social del Uruguay. Montevideo: BCU.

IECON (1971). La distribución del ingreso en Uruguay: Aproximación cuantitativa para el departamento de Montevideo. Santiago de Chile: IECON.

INE (2018). Estimación de la pobreza por el método del ingreso: Año 2017. Montevideo: INE.

ILO (1988). International Standard Classification of Occupations. Ginebra: ILO.

JIMÉNEZ, J. P. (2015). «Introducción». En J. P. JIMÉNEZ (ed.), Desigualdad, concentración del ingreso y tributación sobre las altas rentas en América Latina. Santiago de Chile: CEPAL.

KuSNETZ, S. (1933). «National income», en Encyclopedia of the social sciences, vol. XI. Nueva York: McMillan.

KUSNETZ, S. (1941). National income and its composition: 1919-1938. Nueva York: NBER. LEQUILLER, F. y BLADES, D. (2006). Understanding national accounts. París: OECD.

LóPEZ-CalVa L. y LuSTig, N. (ed.) (2010). Declining inequality in Latina America: A decade of Progress? Virginia: PNUD.

Lustig, N; PEssino, C. y ScotT, J. (eds.) (2013). The impact of taxes and social spending on inequality and poverty in Argentina, Bolivia, Brazil, Mexico, Peru and Uruguay: An overview. CEQ.

MARRoIG, A. y OREIRo, C. (2008). «Determinantes de la distribución del ingreso en Uruguay 1991-2005: Un análisis de microsimulaciones». Quantum, 3(2), 46-63. 
Marshall, A. (1957). Principios de economía: Un tratado de introducción. Madrid: Aguilar.

MARTínEZ, P. (1981). El excedente financiero agropecuario del Uruguay. Montevideo: FCEA.

MARX, K. (1894). Capital, vol. 3. Recuperado de https://www.marxists.org/archive/ marx/works/download/pdf/Capital-Volume-III.pdf.

Medeiros, M.; Ferreira De Souza, P. y Avila De Castro, F. (2015). «0 topo da distribuição de renda no Brasil: Primeiras estimativas com dados tributários e comparação com pesquisas domiciliares (2006-2012)». DADOS-Revista de Ciências Sociais, 58(1), 7-36.

MELGAR, A. (1981a). Aspectos metodológicos sobre la distribución del ingreso en Uruguay. Montevideo: CLAEH.

MElgar, A. (1981b). Distribución del ingreso en el Uruguay. Montevideo: CLAEH.

MELGAR, A. (1995). «Pobreza y distribución del ingreso: La evolución reciente». En CLAEH-PNUD, Salario, pobreza y desarrollo humano en el Uruguay (pp. 87-128). Montevideo: CLAEH.

Melgar, A. y Villalobos, F. (1986). La desigualdad como estrategia: La asignación de recursos en el Uruguay neoliberal. Montevideo: CLAEH.

MoHUn, S. (2016). «Class structure and the US personal income distribution: 1918-2012. Metroeconomica, 67(2), 334-363.

Morley, S. (2000). La distribución del ingreso en América Latina y el Caribe. Santiago de Chile: CEPAL.

MunTANER, C.; LYNCH, J. y OATES, G. (1999). «The social class determinants of income inequality and social cohesion». International Journal of Health Services, 29(4), 699732.

Muinelo, L. y RocA, O. (2017). Long-term effects of fiscal policy in Uruguay. Montevideo: FCEA. (Documentos de Trabajo, 02).

Nolan, B.; SAlverda, W. y SMEEDING, T. (2011). The Oxford handbook of economic inequality. Oxford: Oxford University Press.

Notaro, J. (2016). Procedimiento de cálculo de los ingresos del capital. Uruguay, 20082014. Recuperado de https://www.academia.edu/31907502/PROCEDIMIENTO DE C\%C3\%81LCULO DE LOS INGRESOS DEL CAPITAL. URUGUAY 20082014.

OYHANTÇABAL, G. y SANGUINETTI, M. (2017). «El agro en Uruguay: Renta del suelo, ingreso laboral y ganancias». Problemas del Desarrollo, 48(189), 113-139.

Perazzo, I.; Robino, C. y Vigna, A. (2002). Sistema impositivo y distribución del ingreso en el Uruguay. (Tesis de Licenciatura en Economía). Facultad de Ciencias Económicas y Administración, Montevideo.

PIKETTY, T. (2013). Le capital au XXI siècle. París: Éditions du Seuil. 
PNUD (2008). Desarrollo humano en Uruguay 2008: Política, políticas y desarrollo humano. Montevideo: PNUD.

Portes, A. y Hoffman, K (2003). Las estructuras de clase en América Latina: Composición y cambios durante la época neoliberal. Santiago de Chile: Naciones Unidas.

QUESNAY, F. (1974). «Análisis de la fórmula aritmética del "Tableau Économique" de la distribución de los gastos anuales de una nación agrícola». En V. A. ÁlVAREZ, «Le Tableau Économique» y otros estudios económicos (pp. 197-234). Madrid: Ediciones de la Revista de Trabajo.

QUESNAY, F. (1974). «Máximas generales de la política económica en un país agrícola». En V. A. ÁlVAREZ, «Le Tableau Économique» y otros estudios económicos (pp. 51-82). Madrid: Ediciones de la Revista de Trabajo.

RICARDO, D. (2001). Principles of political economy and taxation. Ontario: Batoche Books. Recuperado de http://socserv.mcmaster.ca/econ/ugcm/3ll3/ricardo/Principles.pdf.

RoLL, E. (1967). Historia de las doctrinas económicas. México: FCE.

Sмith, A. (1904). An inquiry into the nature and causes of the wealth of nations. (edición de E. Cannan). Londres: Methuen \& Co. Recuperado de http://www. econlib.org/library/Smith/smWN.html.

STIGLITZ, J. E. (1969). «Distribution of income and wealth among individuals». Econometrica, 37(3), 382-397.

SZÉKELY, M. y HILGERT, M. (1999). What's behind the inequality we measure: An investigation using Latin American Data. Washington: Inter-American Development Bank.

TERRA, J. P. (1983). Distribución social del ingreso en Uruguay. Montevideo: CLAEH.

Vigorito, A. (1999). Una distribución del ingreso estable: el caso de Uruguay 1986-1997. Montevideo: IECON. (Serie Documentos de Trabajo, 6).

WoDTKE, G. (2014). Class structure and income inequality in the United States. (Ph. D. Thesis). University of Michigan, Michigan.

WOLFF, E. y ZaCHARIAS, A. (2007). Class structure and economic inequality. Nueva York: The Levy Economic Institute.

WulfF GobetTI, S. y ORAIR, R. O. (2017). «Tributação e distribuição da renda no Brasil: Novas evidências a partir das declarações tributárias das pessoas físicas». Brazilian Journal of Political Economy, 37( 2), 267-286. 